\title{
Diagnosis and Management of New Onset Epilepsy in Adults
}

\author{
Anna M Bank and Ruben I Kuzniecky \\ Lenox Hill Hospital/Northwell Health, Zucker School of Medicine at Hofstra University, New York, NY, USA
}

DOI: https://doi.org/10.17925/USN.2020.16.1.32

$\mathrm{O}$ ver the past decade, advances in the diagnosis and treatment of epilepsy have included a new definition of epilepsy, as well as the development of new antiepileptic drugs. Several of these drugs are increasingly prescribed to patients with new onset epilepsy. The aim of this review is to provide clinicians with a framework for diagnosing and managing patients with new onset epilepsy, with an emphasis on selection of an appropriate antiepileptic drug. We begin with the new International League Against Epilepsy definition of epilepsy and its basis in the epilepsy literature, followed by a discussion of the initial work-up of epilepsy. The majority of this review discusses the selection of an appropriate antiepileptic drug for adult patients with new onset epilepsy, including a discussion of the SANAD trials and a systematic review of the data supporting the use of newer agents as monotherapy. Finally, we conclude with a discussion of risk assessment and counseling that should be provided to all patients with a new diagnosis of epilepsy.

\section{Keywords}

Epilepsy, seizures, focal epilepsy, generalized epilepsy, antiepileptic drugs

Disclosures: Anna M Bank is a co-investigator on research projects funded by the Epilepsy Foundation, Xenon Pharmaceuticals, UCB, and Medtronic. Ruben I Kuzniecky receives grant funding from the National Institutes of Health and the Epilepsy Foundation, and research funding from Xenon Pharmaceuticals, UCB, Medtronic, and Eisai.

Review Process: Double-blind peer review.

Compliance with Ethics: This article involves a review of the literature and did not involve any studies with human or animal subjects performed by either of the authors.

Authorship: The named authors meet the International Committee of Medical Journal Editors (ICMJE) criteria for authorship of this manuscript, take responsibility for the integrity of the work as a whole, and have given final approval for the version to be published.

Access: This article is freely accessible at touchNEUROLOGY.com (c) Touch Medical Media 2020

Received: February 7, 2020

Accepted: April 22, 2020

Published Online: July 10, 2020

Citation: US Neurology. 2020;16(1):32-7

Corresponding Author: Anna M Bank

130 East 77th Street, New York, NY 10075, USA.

E: abank@northwell.edu

Support: No funding was received in

the publication of this article.
The International League Against Epilepsy (ILAE) revised its definition of epilepsy in 2014 in order to maximize early identification and treatment of patients with epilepsy. ${ }^{1}$ The ILAE'S conceptual definition of epilepsy, first formulated in 2005 , is "a disorder of the brain characterized by an enduring predisposition to generate epileptic seizures." In practice, this definition corresponded to patients with two or more unprovoked seizures more than 24 hours apart. We know, however, that not all patients with a single unprovoked seizure are equally likely to have a second seizure. Under the new ILAE definition, patients with a single unprovoked seizure and a likelihood of recurrent seizure above $60 \%$ now meet criteria for a diagnosis of epilepsy.

In a systematic review of studies assessing the risk of seizure recurrence following a single unprovoked seizure, between 21 and $45 \%$ of patients had another unprovoked seizure within the first 2 years. ${ }^{2}$ From a practical standpoint, four risk factors have been identified that approximately double a patient's risk of seizure recurrence: a prior brain injury, an abnormal neuroimaging finding that corresponds to the suspected seizure focus, focal or generalized epileptiform discharges on electroencephalogram (EEG), or a nocturnal seizure. ${ }^{3-6}$ Thus, patients with a single unprovoked seizure and at least one of these four risk factors likely has a greater than $60 \%$ chance of recurrent seizure and can be diagnosed with epilepsy (Table 1).

Although a major early study suggested that an abnormal neurologic examination was associated with an increased risk of seizure recurrence, a follow-up study from the same group found no increase in risk. ${ }^{3,7}$ The same follow-up study found that having a sibling with epilepsy increases the risk of recurrence among patients with idiopathic epilepsy, but even among these patients, the recurrence risk is only $46 \%$ at 5 years. Thus, neurologic examination findings and family history of epilepsy should be taken into consideration when evaluating a patient with a first seizure, but these features alone are not sufficient to make a diagnosis of epilepsy.

\section{Consideration of antiepileptic drug treatment in new onset epilepsy} Most early studies of seizure recurrence after an initial unprovoked seizure included both antiepileptic drug (AED)-treated and untreated patients, making it difficult to estimate the true risk of recurrent seizure. The First Seizure Trial Group (FIRST) study found that 51\% of patients who did not start an AED after a first seizure had another one within 2 years, while only $25 \%$ of those who started an AED had another seizure within the same period. ${ }^{8}$ However, later studies have shown that although AED treatment does reduce the risk of seizure recurrence within the first 2 years after an initial seizure, it does not affect the likelihood of long-term seizure remission or quality of life. ${ }^{9,10}$ As early AED treatment is not guaranteed to change a patient's overall outcome, and AEDS are not entirely benign medications, not all patients who are diagnosed with epilepsy choose to start treatment right away. 
Table 1: Diagnosis of new onset epilepsy

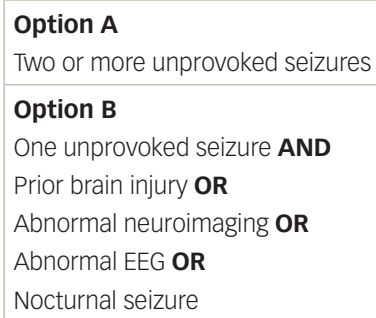

Two or more unprovoked seizures

\section{Option B}

One unprovoked seizure AND

Prior brain injury $\mathbf{O R}$

Abnormal neuroimaging $\mathbf{O R}$

Abnormal EEG OR

Nocturnal seizure

$E E G$ = electroencephalogram.

All decisions about AED treatment should be made in collaboration with the patient after a careful discussion of the risks and benefits, weighing the risk of harm due to seizures against the potential adverse effects of AEDs. The presence of a seizure type that is associated with a higher risk of injury or death, such as tonic-clonic seizures or status epilepticus, may be one factor influencing the patient and physician's decision of whether to start AEDs. ${ }^{11-13}$

\section{Characterization of epilepsy type}

Determination of the patient's epilepsy type-focal or generalized —at the time of initial diagnosis is important because it helps predict prognosis and guide selection of an appropriate AED. This determination is typically made based on seizure semiology along with magnetic resonance imaging (MRI) and EEG findings. Lateralized motor or sensory symptoms, forced eye deviation or head turn, automatisms, language disturbances, and experiential phenomena suggest focal onset, while bilateral myoclonic jerking or initial bilateral tonic activity suggest generalized onset.

However, semiology alone can be misleading: focal seizures may lack lateralizing features at onset, and more than half of patients with generalized epilepsy have focal seizure symptoms. ${ }^{14}$ Moreover, three-quarters of patients with focal epilepsy are amnestic for at least some of their seizures, and 30\% are amnestic for all seizures..$^{15}$ Additionally, up to $60 \%$ of patients do not have an aura preceding their seizures. ${ }^{16}$ These factors make the diagnosis and characterization of epilepsy challenging in many patients.

Most patients with a first seizure should have an MRI, unless there is a contraindication. MRI has a higher yield than computed tomography (CT) for detecting focal epileptogenic lesions..$^{17-19}$ The presence of a focal lesion can confirm a focal onset if the lesion's location corresponds to the patient's semiology. For patients with a clear electroclinical primary generalized epilepsy syndrome, such as juvenile myoclonic epilepsy, neuroimaging may not be required.

If an MRI is obtained, recommended sequences include a 3D T1 sequence with $1 \mathrm{~mm}$ isotropic slices (e.g., magnetization-prepared rapid gradient echo [MPRAGE]), axial and coronal T2 and fluid attenuation inversion recovery (FLAIR) sequences with $\leq 3 \mathrm{~mm}$ slices, and an axial T2* sequence for hemosiderin and calcification (e.g., susceptibilityweighted imaging [SWI] or gradient echo [GRE]). ${ }^{20}$ Contrast is not required for all patients, but should be considered for patients over 50 years of age, due to the higher likelihood of metastatic or primary brain tumor in this age group. When possible, 3 Tesla MRI is preferred over 1.5 Tesla as it is more sensitive for epileptogenic lesions. ${ }^{21,22}$ Recently, 7 Tesla MRI scanners have increased sensitivity even further, but these are typically only available through research protocols. ${ }^{23}$
EEG is the other essential modality for diagnosis and characterization of new onset epilepsy. A single routine EEG may be insufficient for the detection of epileptiform abnormalities; a recent meta-analysis of 15 studies found that the sensitivity of a routine EEG was $17 \% .^{24}$ In order to increase this yield, physicians should consider performing an extended 60-minute EEG, a sleep-deprived EEG, or up to three serial routine EEGs. ${ }^{25-27}$ Ambulatory EEG recordings also increase the likelihood of detecting epileptiform abnormalities; the benefits of prolonged recording diminish after 48 hours, as $95 \%$ of abnormalities are detected within this time frame. ${ }^{28} \mathrm{An}$ inpatient video EEG study may be considered in specific scenarios, such as differentiating epileptic seizures from psychogenic non-epileptic attacks. ${ }^{29}$

\section{Selecting an antiepileptic drug-focal epilepsy The SANAD trial-first-line treatment of new onset focal epilepsy}

The goal of AED treatment in new onset epilepsy is to control seizures with a single AED while minimizing adverse effects. Although there are many drugs available for the treatment of focal epilepsy, there are few controlled studies comparing their efficacy and tolerability. The Standard And New Antiepileptic Drugs (SANAD) trial, published in 2007, was a seminal study of 1,721 patients with focal epilepsy randomized to treatment with carbamazepine, gabapentin, lamotrigine, oxcarbazepine, or topiramate..$^{30}$ The primary outcome was time to treatment failure, defined as discontinuation of the drug due to uncontrolled seizures or adverse effects. Lamotrigine was significantly better than carbamazepine, gabapentin, and topiramate, and non-significantly better than oxcarbazepine. For time to 12-month remission, however, carbamazepine was significantly better than gabapentin and non-significantly better than lamotrigine, topiramate, and oxcarbazepine. ${ }^{30}$

The results of the SANAD trial suggest that lamotrigine is the best first-line treatment for focal epilepsy due to its combination of efficacy and tolerability, with carbamazepine as a reasonable alternative in patients who are able to tolerate it.

\section{Newer antiepileptic drugs for first-line treatment of focal epilepsy}

Since the publication of the SANAD trial, several new focal epilepsy drugs have entered the market. While all of these drugs were initially studied as adjunctive treatments, many are now used as monotherapy, and some, particularly levetiracetam, are now commonly used as first-line treatment. A major advantage of these newer drugs is that the doses can be increased quickly in patients with frequent seizures, without the risk of Stevens-Johnson syndrome, a known adverse effect of lamotrigine, and to a lesser extent carbamazepine.

Among the new AEDs, four-levetiracetam, zonisamide, lacosamide, and eslicarbazepine-have been compared with older AEDs in randomized controlled trials, as shown in Table 2.31-41 Trials were identified using PubMed's clinical trials filter and the search terms "monotherapy," and "[drug name]", and were included if they studied adult patients with focal epilepsy comparing a newer AED to one of the standard AEDs (those included in the SANAD trial).

Of the four new AEDS, levetiracetam is the best studied and most widely prescribed; it has largely become the default first-line AED for new onset epilepsy due to its ease of titration and favorable side effect profile. Levetiracetam is now the most commonly prescribed first-line AED in older 
Table 2: New and standard antiepileptic drugs for monotherapy in focal epilepsy

\begin{tabular}{|c|c|c|c|c|c|}
\hline Drug & Authors, year & $\begin{array}{l}\text { Patients } \\
\text { randomized }\end{array}$ & $\begin{array}{l}\text { Seizure outcome } \\
\text { measure }\end{array}$ & Findings & Comments \\
\hline \multirow[t]{6}{*}{ Levetiracetam } & Brodie et al. $2007^{31}$ & 579 & Seizure freedom & LEV non-inferior to CBZ & \\
\hline & Rosenow et al. $2012^{32}$ & $\begin{array}{l}409 \text { (focal and } \\
\text { generalized) }\end{array}$ & Seizure freedom & LEV equivalent to LTG & $\begin{array}{l}\text { Post-hoc subgroup analysis showed equivalent } \\
\text { recurrence rates for focal and generalized }\end{array}$ \\
\hline & Consoli et al. $2012^{33}$ & 128 (post-stroke) & Seizure freedom & LEV equivalent to CBZ & LEV associated with fewer side effects than CBZ \\
\hline & $\begin{array}{l}\text { Trinka et al. } 2013 \\
(\text { KOMET) }\end{array}$ & $\begin{array}{l}992 \text { (LEV versus } \\
\text { CBZ arm) }\end{array}$ & $\begin{array}{l}\text { Treatment withdrawal, } \\
\text { time to first seizure }\end{array}$ & LEV equivalent to CBZ & $\begin{array}{l}\text { CBZ superior to LEV at } 12 \text { months in patients } \\
\text { with focal seizures only }\end{array}$ \\
\hline & Werhahn et al. $2015^{35}$ & 361 & Seizure freedom & $\begin{array}{l}\text { LEV equivalent to CBZ and } \\
\text { LTG }\end{array}$ & $\begin{array}{l}\text { LEV better than CBZ and equivalent to LTG for } \\
\text { patient retention }\end{array}$ \\
\hline & Kim et al. $2017^{36}$ & 353 & Treatment failure & LEV non-inferior to OXC & \\
\hline Zonisamide & $\begin{array}{l}\text { Baulac et al. 2012,37 } \\
2014^{38}\end{array}$ & 583 & Seizure freedom & ZNS non-inferior to CBZ & \\
\hline Lacosamide & Baulac et al. $2017^{39}$ & 888 & Seizure freedom & LAC non-inferior to CBZ & \\
\hline Eslicarbazepine & Trinka et al. $2018^{40}$ & 815 & Seizure freedom & ESL non-inferior to CBZ & \\
\hline Pregabalin & Kwan et al. $2011^{41}$ & 660 & Seizure freedom & PGB inferior to LTG & \\
\hline
\end{tabular}

$C B Z=$ carbamazepine; $E S L=$ eslicarbazepine; $L A C=$ lacosamide; $L E V=$ levetiracetam; $L T G$ = lamotrigine; $O X C=$ oxcarbazepine; $P G B=$ pregabalin; $Z N S=$ zonisamide.

adults, accounting for $45.5 \%$ of prescriptions. ${ }^{42}$ Studies have shown that levetiracetam has similar efficacy compared to older AEDs, though it was inferior to carbamazepine in a subgroup analysis of one study (Table 2).

Importantly, only two studies compared levetiracetam to lamotrigine; both studies found similar efficacy for the two drugs. ${ }^{32,35}$ The focal epilepsy arm of the SANAD II trial, which will be the largest study to compare the long-term efficacy and tolerability of levetiracetam and lamotrigine, is currently underway (ISRCTN30294119).

Outside of levetiracetam, high-quality data assessing the new AEDs as monotherapy is limited. Zonisamide, lacosamide, and eslicarbazepine have all been shown to be non-inferior to carbamazepine in a single study each (Table 2); zonisamide is also being studied in the focal arm of the SANAD II trial. These three agents can be considered as options for first-line treatment depending on the patient's comorbidities and contraindications. Pregabalin has also been studied in comparison with lamotrigine and was inferior, ${ }^{41}$ and thus is not recommended as first-line treatment.

Brivaracetam was well-tolerated in two randomized studies of conversion from polytherapy to monotherapy, but the number of patients remaining on brivaracetam at the end of these studies was too low to determine its efficacy as monotherapy; ${ }^{43}$ it has also not been studied in direct comparison with older AEDS. Thus, there is insufficient evidence to recommend brivaracetam monotherapy as first-line treatment at this time.

Clobazam, perampanel, and cenobamate are new AEDs with the potential for use as monotherapy. Both clobazam and perampanel were effective and well-tolerated in small retrospective studies that included patients with both focal and generalized epilepsy.44,45 Clobazam was also superior to carbamazepine and phenytoin in a randomized trial of pediatric patients, but has not been directly compared to the older AEDs in an adult population. ${ }^{46}$ Cenobamate, an enhancer of fast and slow sodium channel inactivation with once-daily dosing, recently demonstrated efficacy as an adjunctive agent for patients with focal epilepsy, but has not yet been studied as monotherapy. ${ }^{47}$

\section{Summary of first-line treatment for focal epilepsy}

Levetiracetam has performed as well as, or slightly worse than, older AEDs in head-to-head trials, ${ }^{31-36}$ and is a reasonable first-line treatment in patients without a history of psychiatric issues, particularly if seizures are frequent or patients have difficulty with the lamotrigine titration schedule. In patients with psychiatric comorbidities, we recommend lamotrigine as first-line treatment. The pending SANAD II trial will more definitively answer the question of which of these two AEDs is superior with regards to both efficacy and tolerability. Depending on the patient's comorbidities and side effect tolerance, several of the older AEDs (carbamazepine, oxcarbazepine, topiramate) and newer AEDs (zonisamide, lacosamide, eslicarbazepine) may be reasonable alternatives. Brivaracetam, clobazam, perampanel, and cenobamate may be viable options in the future, but there is insufficient evidence at this time. Gabapentin and pregabalin should not be used as first-line treatments.

\section{Selecting an antiepileptic drug-generalized or unclassified epilepsy \\ The SANAD trial-first-line treatment of new onset generalized epilepsy}

The 2007 SANAD trial included a second arm, which studied patients with generalized epilepsy or epilepsy that could not be definitively classified at the time of treatment initiation. ${ }^{48}$ Valproate was significantly better than topiramate for time to treatment failure in the overall analysis, and significantly better than both topiramate and lamotrigine in patients with a confirmed diagnosis of generalized epilepsy. For time to 12-month remission, valproate was significantly better than lamotrigine in both groups, but not significantly different from topiramate in either group. Thus, valproate appeared to have the best combination of efficacy and tolerability of the three drugs studied. 
Despite valproate's success in SANAD, its use outside of the trial setting is problematic because of its adverse effects. Among women of childbearing age, valproate is contraindicated due to teratogenicity, as it significantly increases the risk of congenital malformations and long-term neurocognitive deficits. ${ }^{49-52}$ As of 2018, the European Union has banned the use of valproate in women of childbearing age unless they are enrolled in a pregnancy prevention program..$^{53}$ Valproate also has several other undesirable side effects including weight gain, hair loss, polycystic ovarian syndrome, and hepatic encephalopathy. ${ }^{54-57}$ Alternative first-line treatments are needed, especially for women of childbearing age.

\section{Newer alternatives to valproate for the treatment of generalized epilepsy}

Levetiracetam is the most extensively studied new AED for generalized epilepsy. Two of the monotherapy trials described above also included patients with generalized epilepsy. ${ }^{32,34}$ In the KOMET study, levetiracetam was equivalent to valproate for both treatment withdrawal rate and time

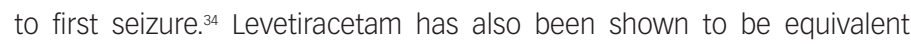
to lamotrigine for seizure freedom rate in a heterogenous sample; post-hoc subgroup analysis showed that this was true for both focal and generalized epilepsy. ${ }^{32}$

Recently, Marson and colleagues presented the results of the generalized epilepsy arm of the SANAD II trial. ${ }^{58}$ Valproate was superior to levetiracetam for time to treatment failure, time to first seizure, and time to 24-month remission. Interestingly, the difference in time to treatment failure was attributable to inadequate seizure control, rather than to adverse effects, which suggests that valproate was not more poorly tolerated than levetiracetam in this sample.

As noted above, both clobazam and perampanel were shown to have good efficacy and tolerability in retrospective observational studies that included patients with generalized epilepsy, but neither drug has been compared with any of the older drugs in a randomized trial. ${ }^{44,45}$ To our knowledge, none of the other new AEDs have been studied as monotherapy in patients with generalized epilepsy.

\section{Summary of first-line treatment for generalized and unclassified epilepsy}

Although the SANAD I| results suggest that valproate remains the most effective AED for generalized or unclassified epilepsy, we do not recommend it as first-line treatment due to its adverse effects. Lamotrigine is a good choice if seizure frequency is low enough to allow slow dose increases. If rapid onset is needed, levetiracetam can be used. If seizures are refractory to these two agents, valproate can be used after careful discussion of the risks and benefits.

\section{Counseling patients with new onset epilepsy}

Patients with a new diagnosis of epilepsy typically have many questions about the disease and its impact on their lives. The most frequent questions are about the long-term prognosis of epilepsy, whether to start AED treatment, and whether AED treatment will be lifelong. As discussed above, patients should be informed that immediate AED treatment reduces the risk of early seizure recurrence, but does not change the overall prognosis of epilepsy, and the decision about whether to start an AED should be made on an individualized basis. Patients should be informed that about half of patients will achieve seizure control with their first AED, while an additional $20 \%$ will respond to a second or third drug. ${ }^{59}$ Additionally, they should be aware that any attempts to discontinue AED treatment should not be initiated until after at least 2 years of seizure freedom, and that nearly half of patients will have a recurrence of seizures after AED withdrawal. ${ }^{60}$

Outside of seizure control, the most common practical questions relate to driving. Any seizure with impaired awareness-absence, tonic-clonic, or focal unaware - can cause significant harm if it occurs while the patient is driving. Most states require an interval of seizure freedom before a patient can resume driving. Physicians should ensure that patients are familiar with their state's laws. Some states require physicians to report their patients with epilepsy or seizures to the Department of Motor Vehicles, while others do not. ${ }^{11}$ It is important to note that in states without mandatory reporting, physicians may not have legal protection when disclosing a diagnosis of epilepsy without the patient's consent. In these cases, physicians will need to weigh the risks of the patient continuing to drive (particularly in the case of commercial drivers) against the risks of legal ramifications of violating the patient's confidentiality. All conversations about driving should be documented in the patient's medical record.

Women of childbearing age should additionally be counseled about the implications of epilepsy and AEDs on pregnancy. Patients should be aware that seizures, especially generalized tonic-clonic seizures, and some AEDS, can have adverse effects on a developing fetus. In women who are not planning pregnancy, long-acting contraception with an intrauterine device or depot injection is preferred as these minimize opportunity for user error. Patients should also know that treatment with enzyme-inducing AEDs can lower the efficacy of hormonal contraception..$^{22}$ All women with epilepsy who are planning pregnancy should inform their neurologist ahead of time; if a pregnancy is unplanned, the neurologist should be informed as soon as possible. Additionally, all women with epilepsy of childbearing age should take folic acid whether or not a pregnancy is planned, as this reduces the risk of neural tube defects that develop early in the first trimester, often before a pregnancy is known. ${ }^{63}$

The most difficult topic to discuss with newly diagnosed patients is sudden unexpected death in epilepsy (SUDEP). Risk factors for SUDEP include high seizure frequency, early age of onset, long duration of disease, generalized tonic-clonic seizures, nocturnal seizures, living alone, male gender, alcohol dependence, and substance abuse. ${ }^{64-66}$ Most neurologists believe that all patients with epilepsy should be informed about SUDEP, while some argue that SUDEP counseling is only necessary for patients who are at high risk. ${ }^{67}$ We recommend discussing SUDEP with all patients with new onset epilepsy as part of an initial discussion about the importance of medication compliance, with more frequent and extensive counseling for high-risk patients. $\square$ 


\section{Take-home messages}

- Patients should be diagnosed with epilepsy if they have two or more unprovoked seizures, or a single unprovoked seizure and an abnormal MRI, epileptiform abnormalities on electroencephalogram (EEG), a history of head trauma, or a nocturnal seizure.

- Work-up for new onset epilepsy should include magnetic resonance imaging, ideally 3 Tesla, and an EEG, with the aim of classifying the disease as focal or generalized. If the initial routine outpatient EEG is normal, options to consider include an extended routine EEG, serial routine EEGs, a sleep-deprived EEG, or an ambulatory EEG.

- Lamotrigine is the recommended first-line treatment for focal epilepsy in most patients, but levetiracetam is a reasonable alternative; a large trial comparing the two is currently underway.

- While recent evidence suggests that valproate is the most effective treatment for generalized or unclassifiable epilepsy, it is contraindicated in women of childbearing age and poorly tolerated in many other patients; lamotrigine and levetiracetam are better-tolerated initial treatments for generalized or unclassifiable epilepsy.

- All patients with a new diagnosis of epilepsy should be counseled about epilepsy prognosis, driving restrictions, implications for childbearing, and SUDEP.

1. Fisher RS, Acevedo C, Arzimanoglou A, et al. ILAE official report: a practical clinical definition of epilepsy. Epilepsia. 2014;55:475-82

2. Krumholz A, Wiebe S, Gronseth GS, et al. Evidence-based guideline: Management of an unprovoked first seizure in adults: report of the Guideline Development Subcommittee of the American Academy of Neurology and the American Epilepsy Society. Epilepsy Curr. 2015;15:144-52.

3. Hauser WA, Rich SS, Annegers JF, et al. Seizure recurrence after a 1st unprovoked seizure: an extended follow-up. Neurology. 1990;40:1163-70

4. Chen T, Si Y, Chen D, et al. The value of 24-hour video-EEG in evaluating recurrence risk following a first unprovoked seizure: a prospective study. Seizure. 2016;40:46-51.

5. Kho LK, Lawn ND, Dunne JW, Linto J. First seizure presentation: do multiple seizures within 24 hours predict recurrence? Neurology 2006:67:1047-9.

6. Bora I, Seçkin B, Zarifoglu M, et al. Risk of recurrence after first unprovoked tonic-clonic seizure in adults. J Neurol. 1995;242:157-63

7. Annegers JF, Shirts SB, Hauser WA, et al. Risk of recurrence after an initial unprovoked seizure. Epilepsia. 1986;27:43-50.

8. First Seizure Trial Group (FIR.S.T. Group). Randomized clinical trial on the efficacy of antiepileptic drugs in reducing the risk of relapse after a first unprovoked tonic-clonic seizure. Neurology 1993:43:478-83.

9. Marson A, Jacoby A, Johnson A, et al. Immediate versus deferred antiepileptic drug treatment for early epilepsy and single seizures: a randomised controlled trial. Lancet. 2005;365:2007-13.

10. Leone MA, Solari A, Beghi E. Treatment of the first tonic-clonic seizure does not affect long-term remission of epilepsy. Neurology. 2006;67:2227-9.

11. Willems $L M$, Watermann $N$, Richter $S$, et al. Incidence, risk factors, and consequences of epilepsy-related injuries and accidents: a retrospective, single-center study. Front Neurol. 2018;9:414.

12. Lhatoo SD, Johnson AL, Goodridge DM, et al. Mortality in epilepsy in the first 11 to 14 years after diagnosis: multivariate analysis of a long-term, prospective, population-based cohort. Ann Neurol. 2001;49:336-44.

13. Neligan A, Shorvon SD. Frequency and prognosis of convulsive status epilepticus of different causes. JAMA Neurol. 2010;67:931-40.

14. Seneviratne $\mathrm{U}$, Woo JJ, Boston RC, et al. Focal seizure symptoms in idiopathic generalized epilepsies. Neurology. 2015;85:589-95.

5. Blum DE, Eskola J, Bortz JJ. Patient awareness of seizures Neurology. 1996;47:260-4.

16. Nakken KO, Solaas MH, Kjeldsen MJ, et al. The occurrence and characteristics of auras in a large epilepsy cohort. Acta Neurol scand. 2009;119:88-93.

17. Sperling MR, Wilson G, Engel J Jr, et al. Magnetic resonance imaging in intractable partial epilepsy: correlative studies. Ann Neurol. 1986;20:57-62.

18. Heinz ER, Heinz TR, Radtke R, et al. Efficacy or MR vS CT in epilepsy. AJR Am J Roentgenol. 1989;152:347-52.

19. Olszewska DA, Costello DJ. Assessment of the usefulness of magnetic resonance brain imaging in patients presenting with acute seizures. Ir J Med Sci. 2014;183:621-4.

20. Wellmer J, Quesada CM, Rothe L, et al. Proposal for a magnetic resonance imaging protocol for the detection of epileptogenic lesions at early outpatient stages. Epilepsia. 2013;54:1977-87.

21. Knake S, Triantafyllou C, Wald LL, et al. 3T phased array MR improves the presurgical evaluation in focal epilepsies: a prospective study. Neurology. 2005;65:1026-31.

22. Phal PM, Usmanov A, Nesbit GM, et al. Qualitative comparison of 3-T and 1.5-T MRI in the evaluation of epilepsy. AJR Am J Roentgenol. 2008:191:890-5.

23. De Ciantis A, Barba C, Tassi L, et al. 7T MRI in focal epilepsy with unrevealing conventional field strength imaging. Epilepsia. 2016;57:445-54

24. Bouma HK, Labos C, Gore GC, et al. The diagnostic accuracy of routine electroencephalopgraphy after a first unprovoked seizure Eur J Neurol. 2016:23:455-63.

25. Burkholder DB, Britton JW, Rajasekaran V, et al. Routine vs extended outpatient EEG for the detection of interictal epileptiform discharges. Neurology. 2016;86:1524-30.

26. Geut I, Weenink S, Knottnerus ILH, et al. Detecting interictal discharges in first seizure patients: ambulatory EEG or EEG after sleep deprivation? Seizure. 2017:51:52-4.

27. Baldin E, Hauser WA, Bucchalter JR, et al. Yield of epileptiform electroencephalogram abnormalities in incident unprovoked seizures: a population-based study. Epilepsia. 2014;55:1389-98.

28. Faulkner HJ, Arima H, Mohamed A. Latency to first interictal epileptiform discharge in epilepsy with outpatient ambulatory EEG. Clin Neurophysiol. 2012;123:1732-5.

29. LaFrance WC Jr, Baker GA, Duncan R, et al. Minimum requirements for the diagnosis of psychogenic nonepileptic seizures: a staged approach: a report from the International seizures: a staged approach: a report from the International League Against Epilepsy Non
Epilepsia. 2013;54:2005-18.

30. Marson AG, Al-Kharusi AM, Alwaidh M, et al. The SANAD study of effectiveness of carbamazepine, gabapentin, lamotrigine, oxcarbazepine, or topiramate for treatment of partial epilepsy: an unblended randomised controlled trial. Lancet. 2007:369:10-15.

31. Brodie MJ, Perucca E, Ryvlin P, et al. Comparison of levetiracetam and controlled-release carbamazepine in newly diagnosed epilepsy. Neurology. 2007;68:402-8.

32. Rosenow F, Schade-Brittinger C, Burchardi N, et al. The LaLiMo Trial: lamotrigine compared with levetiracetam in the initial 26 weeks of monotherapy for focal and generalised epilepsy -an open-label, prospective, randomised controlled multicenter study. J Neurol Neurosurg Psychiatry. 2012;83:1093-8.

33. Consoli D, Bosco D, Postorino P, et al. Levetiracetam versus carbamazepine in patients with late poststroke seizures: a multicenter prospective randomised open-label study (EpIC Project). Cerebrovasc Dis. 2012;34:282-9.

34. Trinka E, Marson AG, Van Paesschen W, et al. KOMET: an unblinded, randomised, two parallel-group, stratified trial comparing the effectiveness of levetiracetam with controlled-release carbamazepine and extended-release sodium valproate as monotherapy in patients with newly diagnosed epilepsy. J Neurol Neurosurg Psychiatry. 2013:84:1138-47.

35. Werhahn KJ, Trinka E, Dobesberger J, et al. A randomised, double-blind comparison of antiepileptic drug treatment in the elderly with new-onset focal epilepsy. Epilepsia. 2015;56:450-9.

36. Kim JH, Lee SK, Loesch C, et al. Comparison of levetiracetam and oxcarbazepine monotherapy among Korean patients with newly diagnosed focal epilepsy: a long-term, randomized, open-labe trial. Epilepsia. 2017:58:e70-4.

37. Baulac M, Brodie MJ, Patten A, et al. Efficacy and tolerability of zonisamide versus controlled-release carbamazepine for newly diagnosed partial epilepsy: a phase 3, randomised, double-blind non-inferiority trial. Lancet Neurol. 2012;11:579-88.

38. Baulac, Patten A, Giorgi L. Long-term safety and efficacy of zonisamide versus carbamazepine monotherapy for treatment of partial seizures in adults with newly diagnosed epilepsy: results of a phase III, randomized, double-blind study. Epilepsia. 2014:55:1534-43.

39. Baulac M, Rosenow F, Toledo M, et al. Efficacy, safety, tolerability of lacosamide monotherapy versus controlled-release carbamazepine in patients with newly diagnosed epilepsy: a phase 3 , randomized, double-blind non-inferiority trial. Lancet Neurol. 2017;16:43-54.

40. Trinka E, Ben-Menachem E, Kowacs PA, et al. Efficacy and safety of eslicarbazepine acetate versus controlled-release carbamazepine monotherapy in newly diagnosed epilepsy: a phase III double-blind, randomized, parallel-group, multicenter a phase III double-blind, randomized,
study. Epilepsia. 2018;59:479-91.

41. Kwan P, Brodie MJ, Kalviainen R, et al. Efficacy and safety of pregabalin versus lamotrigine in patients with newly diagnosed partial seizures: a phase 3, double-blind, randomised, parallel-group trial. Lancet Neurol. 2011:10:881-90.

42. Martin RC, Faught E, Szaflarski JP, et al. What does the US Medicare administrative claims database tell us about the initia antiepileptic drug treatment for older adults with new-onset epilepsy? Epilepsia. 2017;58:548-57.

43. Arnold S, Badalamenti V, Diaz A, et al. Conversion to brivaracetam monotherapy for the treatment of patients with focal seizures: two double-blind, randomized, multicenter, historical control, phase III studies. Epilepsy Res. 2018;141:73-82.

44. Mehndiratta MM, Krishnamurthy M, Rajesh KN, et al. Clobazam monotherapy in drug naive adult patients with epilepsy. Seizure. 2003:12:226-8.

45. Gil-Nagel A, Burd S, Toledo M, et al. A retrospective, multicente study of perampanel given as monotherapy in routine clinical care in people with epilepsy. Seizure. 2018;54:61-6.

46. Canadian Study Group for Childhood Epilepsy. Clobazam has equivalent efficacy to carbamazepine and phenytoin as monotherapy for childhood epilepsy. Epilepsia. 1998;39:952-9.

47. Krauss GL, Klein P, Brandt C, et al. Safety and efficacy of adjunctive cenobamate (YKP3089) in patients with uncontrolled focal seizures: a multicentre, double-blind, randomised, placebo-controlled, dose-response trial. Lancet Neurol. 2020;19:38-48

48. Marson AG, Al-Kharusi AM, Alwaidh M, et al. The SANAD study of effectiveness of valproate, lamotrigine, or topiramate for generalised and unclassifiable epilepsy: an unblinded randomised controlled trial. Lancet. 2007:369:1016-26.

49. Jentink J, Loane MA, Dolk H, et al. Valproic acid monotherapy in pregnancy and major congenital malformations. N Eng/ I Med. 2010;362:2185-93.

50. Meador KJ, Baker GA, Browning N, et al. Cognitive function at 3 years of age after fetal exposure to antiepileptic drugs. N Eng/ J Med. 2009;360:1597-605.

51. Meador KJ, Baker GA, Browning N, et al. Effects of fetal antiepileptic drug exposure: outcomes at age 4.5 years Neurology. 2012;78:1207-14.

52. Meador KJ, Baker GA, Browning N. et al. Fetal antiepileptic drug exposure and cognitive outcomes at age 6 years (NEAD study): a prospective observational study. Lancet Neurol. 2013;12:244-52

53. European Medicines Agency. New measures to avoid valproate exposure in pregnancy endorsed. 2018. Available at: www.ema europa.eu/en/news/new-measures-avoid-valproate-exposurepregnancy-endorsed (accessed June 12, 2020).

54. Petty SJ, Kantor S, Lawrence KM, et al. Weight and fat distribution in patients taking valproate: a valproate-discordant gender-matched twin and sibling pair study. Epilepsia. 2014;55:1551-7.

55. Chen B, Choi H, Hirsch $L$, et al. Cosmetic side effects of antiepileptic drugs in adults with epilepsy. Epilepsy Behav. 2015;42:129-37

56. Hu X, Wang J, Dong W, et al. A meta-analysis of polycystic ovary syndrome in women taking valproate for epilepsy. Epilepsy Res. 2011;97:73-82.

57. Chopra A, Kolla BP, Mansukhani MP, et al. Valproate-induced hyperammonemic encephalopathy: an update on risk factors, clinical correlates, and management. Gen Hosp Psychiatry. 2012;34:290-8.

58. Marson A, Burnside G, Appleton R, et al. The SANAD II study of effectiveness of valproate or levetiracetam in generalised and unclassifiable epilepsy: an unblinded randomized controlled trial. Presented at: 33rd International Epilepsy Congress, Bangkok, Thailand, June 22-26, 2019. Abstract 1027.

59. Kwan P, Brodie MJ. Early identification of refractory epilepsy. N Eng/ J Med. 2000;342:314-9.

60. Practice parameter: a guideline for discontinuing antiepileptic drugs in seizure-free patients-summary statement. Report of the Quality Standards Subcommittee of the American Academy of Neurology. Neurology. 1996;47;600-2 
61. Berger JT, Rosner F, Kark P, et al. Reporting by physicians of impaired drivers and potentially impaired drivers. The Committee on Bioethical Issues of the Medical Society of the State of New York. J Gen Intern Med. 2000;15:667-72.

62. Gooneratne IK, Wimalaratna M, Ranaweera AKP et al. Contraception advice for women with epilepsy. BMJ. 2017;11:357.

63. Harden CL, Pennell PB, Koppel BS, et al. Practice parameter update: Management issues for women with epilepsy-Focus on pregnancy (an evidence-based review): vitamin K, folic acid, blood levels, and breastfeeding. Neurology. 2009;73:142-9.

64. Tomson T, Walczak T, Sillanpaa M, et al. Sudden unexpected death in epilepsy: a review of incidence and risk factors. Epilepsia. 2005;46:54-61.

65. Sveinsson $\mathrm{O}$, Andersson $\mathrm{T}$, Carlsson $\mathrm{S}$, et al. The incidence of SUDEP: a nationwide population-based cohort study. Neurology. 2017;89:170-7
66. Sveinsson O, Andersson T, Mattsson P, et al. Clinical risk factors in SUDEP: a nationwide population-based case-control study. Neurology. 2020;94:e419-29.

67. Brodie MJ, Holmes GL. Should all patients be told about sudden unexpected death in epilepsy (SUDEP)? Pros and Cons. Epilepsia. 2008;49:99-101. 